\title{
ANALYTICAL STUDY AND IMPLEMENTATION OF DIGITAL EXCITATION SYSTEM FOR DIESEL GENERATORS
}

\author{
Sreehari $\mathbf{S}^{1}$, Deepak $\mathbf{C} \mathbf{R}^{2}$ \\ ${ }^{1}$ Student, Electrical and Electronics Department, ${ }^{2}$ Student, Computer Science Department, \\ Amrita Vishwa Vidyapeetham, Tamil Nadu, India, sreeharicybex@gmail.com, deepakcrk@gmail.com
}

\begin{abstract}
This paper aims at the study of DIGITAL EXCITATION SYSTEM [Micro Controller based automatic voltage regulator] employed for synchronous generators. The main component of the digital excitation system is the Automatic Voltage Regulator. This maintains the machine terminal voltage in desired reference, which can be varied in order to accomplish different power system requirements .The digital excitation system is developed from the digital signal processor whichwill capture of variables, performs mathematical operations and control algorithms and sends the control signals to the actuator. Automatic Voltage regulator (automatic/manual control), over excitation limiter, Under excitation limite and volts/hertz limiter are included in this digital excitation system. The exciter is controlled three phase SCR's bridge
\end{abstract}

Index Terms: DIGITAL EXCITATION SYSTEM, Automatic Voltage Regulator, SCR Bridge, synchronous generator,dsPIC30F4012.

\section{INTRODUCTION}

Microcontroller based automatic voltage regulator is used to maintain the terminal voltage of the alternator constant from no load to full load irrespective of power factor and frequency. As the terminal voltage decreases depending on machine reactance and load power factor. A suitable excitation control is required to keep Alternator terminal voltage constant.Industrial loads are always fluctuating and controlling the excitation is difficult by manual operation. So suitable automatic voltage regulator is required to change excitation at a faster rate against quick changing loads.

Digital Excitation system panel senses the alternator terminals voltage, varies the power fed to the alternator field to keep terminal voltage constant irrespective of load conditions. This is achieved by thyristor controlled rectifier where triggering phase angle of the thyristor is varied by fast acting highly sensitive solid state control circuit which works on close loop principle.

The field of the alternator is fed from AC exciter and rotating rectifier. The AC exciter field is controlled from AVR panel. The AVR panel is having auto and manual channel. They have separate control circuit, whereas power circuit is common, hence when auto channel is workingit can be transformed to manual channel without disturbing the load. The manual channel is controlled by follow up circuit while system is operating in auto channel. The follow up unit sense the output reference of auto channel and gives signal to Digital Reference Unit (DRU) of the manual channel to maintain the same reference of auto channel.The power circuit consists of half controlled full wave thyristor rectifier bridge. The dsPIC30F4012 will control the firing angle of the thyristors which control the power fed to the field, overexcited as well as the under excited condition of the generator by adjusting the firing angle. So, the software embedded in the microcontroller helps to maintain fixed terminal voltage, logical operations of the generator, protection of generator and to maintain the constant power factor by adjusting the firing angle of the thyristors.

\section{SYNCHRONOUS GENERATOR}

\subsection{PRINCIPLE OF OPERATION}

A synchronous generator is universally employed for the generation of three phase power at all generating stations. The largest-sized electrical machines are polyphase synchronous generators. Under steady-state conditions, the operating speed of a synchronous machine depends on the frequency ofarmature currents and the number of field poles and is given by

$$
F=\frac{N P}{120}
$$

Where,

$\mathrm{P}=$ number of field poles

$\mathrm{N}=$ rotor speed in $\mathrm{rpm}$ (Synchronous speed)

$\mathrm{F}=$ frequency of armature currents. 

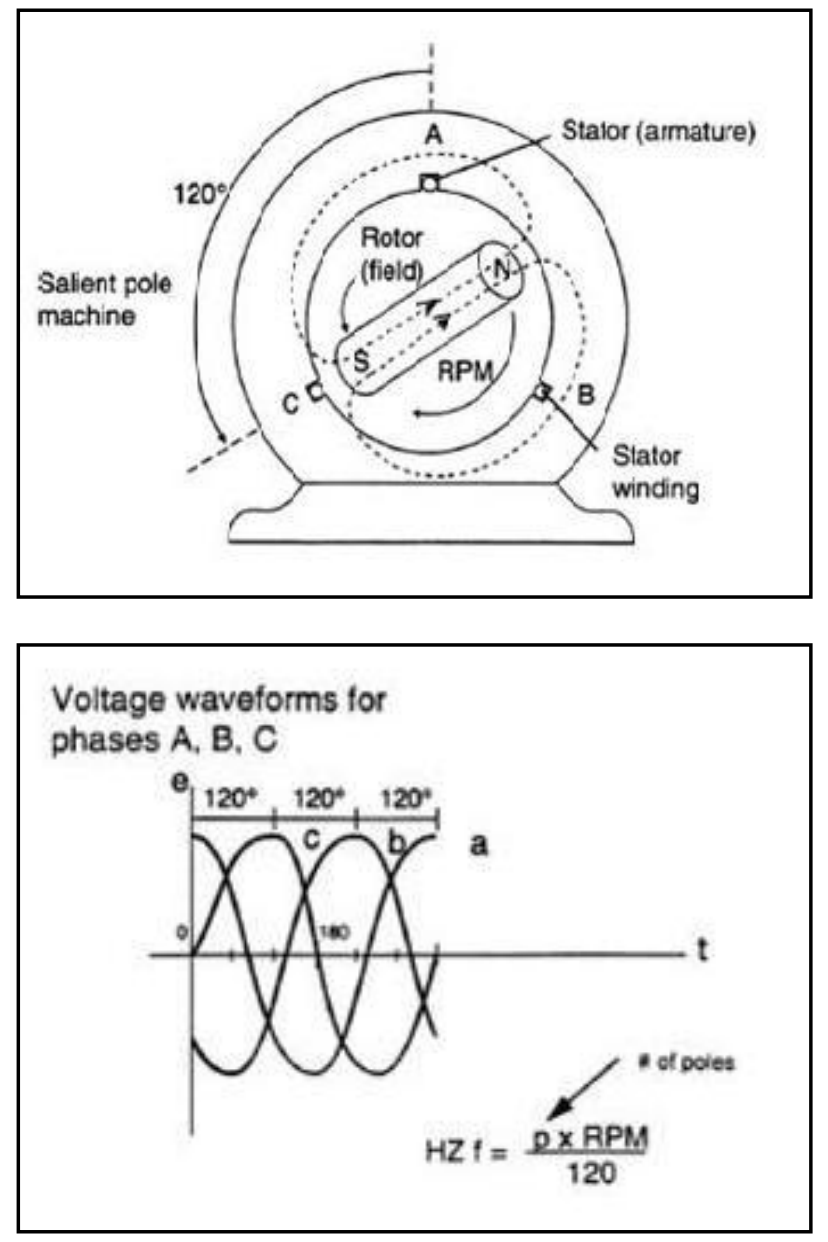

Fig.1. Synchronous machine construction and three phase voltage generated at the terminals of generator

For the successful operation, the rotational speed of the synchronous machine must synchronize with the frequency of the energy system that meansthe stator flux and rotor flux should get tightly bounded and the value of slip should be zero. For production of torque the relative velocity between the interacting stator and rotor fields must be zero.

When a synchronous generator is excited with field current and driven at constant speed, balanced voltage is generated in the armature winding. If balanced load is connected across armature winding, balanced armature current at the same frequency as the e.m.f will flow. Since the frequency of generated e.m.f is related to the rotor speed, while the speed of the armature rotating m.m.f is related to the frequency of the current, it follows that the armature m.m.f rotates synchronously with the rotor field. An increase in rotor speed results in a rise in the frequency of e.m.f and current, while the power factor is determined by the nature of the load.
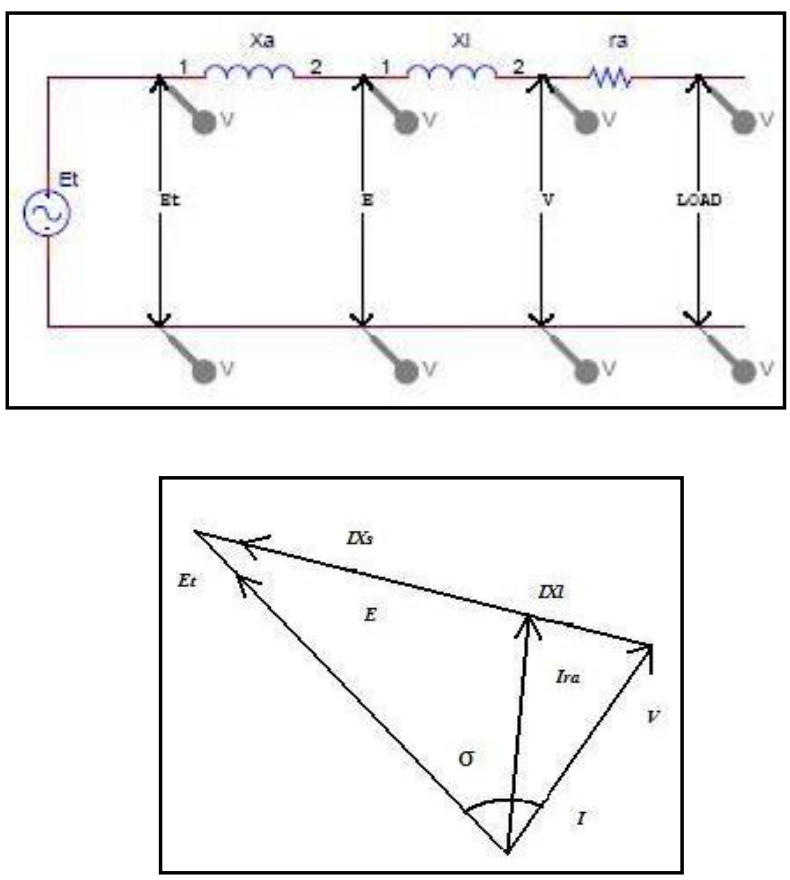

Fig.2a. Equivalent circuit and phasor diagram for R load

Since the armature mmf rotates at the same speed as the main field, it produces a corresponding e.m.f in the armature winding. The effects of armature reaction and armature winding leakage are considered to produce an equivalent internal voltage drop across the synchronous reactance Xs, while the field excitation is accounted for by the open-circuit armature voltage Ef. The impedance $\mathrm{Zs}=(\mathrm{R}+\mathrm{j} \mathrm{Xs})$ is known as the synchronous impedance of the synchronous generator, where $\mathrm{R}$ is the armature resistance.

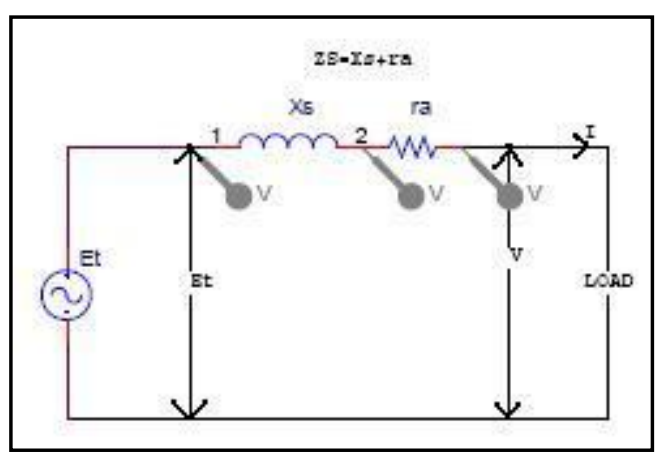

For steady-state performance analysis, the per-phase equivalent circuit shown in Figure 2 is used.

The synchronous generator, under the assumption of constant synchronous reactance, may be considered as represent able by an equivalent circuit comprising an ideal 


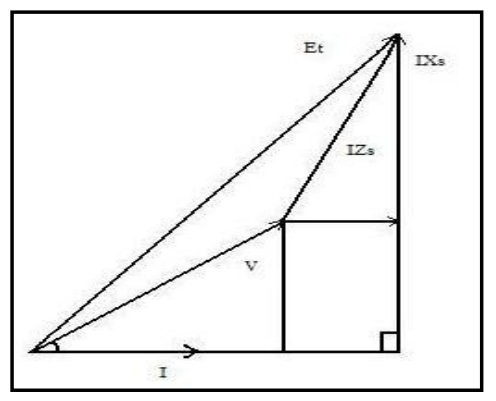

Fig.2b. Equivalent circuit and phasor diagram for R-L load

Winding in which an e.m.f. Et proportional to the field excitation is developed, the winding being connected to the terminals of the machine through a resistance ra and reactance $(\mathrm{Xl}+\mathrm{Xa})=\mathrm{Xs}$ all per phase. This is shown in Fig.2. The principal characteristics of the synchronous generator will be obtained qualitatively from this circuit $[1,3,6]$.

\subsection{GENERATOR LOAD CHARACTERISTICS}

On open circuit the terminal voltage $\mathrm{V}$ is the same as the open circuit e.m.f. Et .Suppose a unity-power-factor load be connected to the machine. The flow of load current produces a voltage drop IZs in the synchronous impedance and terminal voltage $\mathrm{V}$ is reduced. The terminal voltage is obtained from the complex summation

$$
V=E_{t}-I Z_{S}
$$

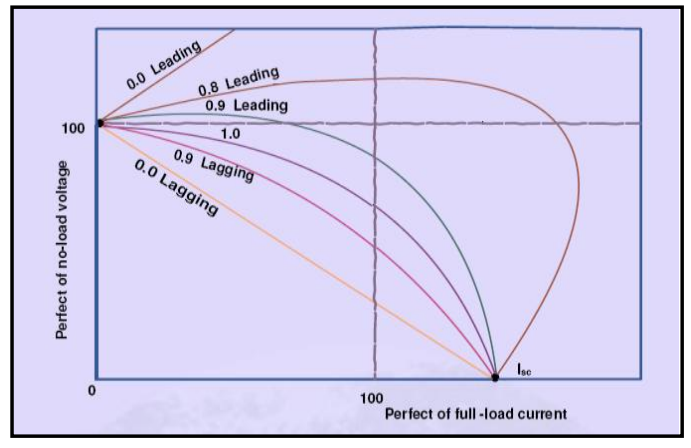

Fig.2c. Generator load characteristics

Algebraically this can be written

$$
V=\sqrt{\left(E_{t}^{2}-I^{2} X_{s}^{2}\right)}-I_{r}
$$

for non-reactive loads. Since normally $r$ is small compared with Xs

$$
V^{2}+I^{2} X_{s}^{2} \approx E_{t}^{2}=\text { const }
$$

so that the V/I curve is as given below[6].

\subsection{GENERATOR EXCITATION FOR CONSTANT VOLTAGE}

Since the e.m.f. Et is proportional to the excitation when the synchronous reactance is constant, the Eqn.

$$
E_{t}^{2}=(V \cos \phi+I r)^{2}+(V \sin \phi+I X s)^{2}
$$

can be applied directly to obtain the excitation necessary to maintain constant output voltage for all loads. All unity-and lagging power-factor loads will require an increase of excitation with increase of load current, as a corollary of Fig.3. Low-leading-power-factor loads, on the other hand, will require the excitation to be reduced on account of the direct magnetizing effect of the zero- power-factor component.

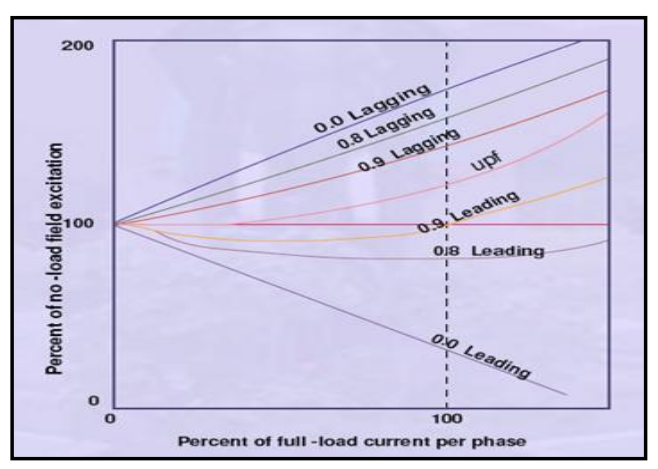

Fig.3. Generator excitation for constant voltage

The voltage-regulation of a synchronous generator is the voltage rise at the terminals when a given load is thrown off, the excitation and speed remaining constant.The voltage-rise is clearly the numerical difference between Et and V, where V is the terminal voltage for a given load and Et is the opencircuit voltage for the same field excitation.Expressed as a fraction, the regulation is[6]

$$
\varepsilon=\frac{\left(E_{t}-V\right)}{V} \text { perunit }
$$

\section{PROPOSED SCHEME:MICROCONTROLLER BASED AUTOMATIC VOLTAGE REGULATOR}

As the automatic voltage regulator (AVR), which regulates generator voltage, is a device indispensable for operation, it is required to have superior reliability in addition to easy maintenance or repair features. And, recently there exists an ever-increasing demand for improved system stability through the excitation control (AVR) in order to prevent decline in system stability in line with the increase in power system and power re-routing. At the same time, digital devices as represented by micro-controllers have been making a remarkable progress. The digital excitation systems are achieving, high reliability, easy-operation, and easy 
maintainability plus improved space factor. The main components of the digital excitation system include:

a] A.C.EXCITATION UNIT

b] ROTATING DIODE or ROTATING RECTIFIER

c] MAIN SYNCHRONOUS GENERATOR

d] MICROCONTROLLER UNIT [dsPIC30F4012]

e] 3-PHASE FULLY BRIDGED THYRISTOR BRIDE

f] ZEROCROSSING DETECTOR

The digital automatic voltage regulator is needed to control the voltage and the reactive power of the generator. The main tasks of the regulator are to control the voltage in the steady state, regulate the voltage under fault conditions and to facilitate reactive power load-sharing between generators in parallel operation. The voltage control is used in generators in island mode operation or in parallel operation with weak networks. Power factor is controlled in case the generator is operating in parallel with a strong network. In case of voltage control, the reactive power sharing between generators can be controlled additionally by using a reactive droop compensation method in which the voltage is lowered in the generator from which the reactive current flows. This lowers the need for excitation and thus also the reactive current. The simplest analog automatic voltage-regulator solutions only control the excitation current in load operation. In more complex digital regulators, the control functions usually include power factor control, generator soft start capability, over-voltage and under-frequency protection, under- and over excitation current limitation and voltage matching before synchronizing. The D-AVR supplies and controls the current in the field winding of the exciter to provide the needed excitation current to the field winding in the rotor. The excitation is controlled typically by means of a PID control algorithm and a diode-bridge rectifier fed switch-mode power supply or a thyristor-bridge rectifier. The diode bridge rectifier is a line-commutated converter in which the firing of the diodes depends only on the line voltage. The Thyristor Bridge is a self-commutated converter, and the thyristors must be fired to conduct the current. Typically, Digital automatic voltage regulators can be supplied by either single or three phase AC voltage or DC voltage. However, the excitation current output from the regulator may be limited to a value lower than the rated value when supplied with single-phase voltage. A higher current can be achieved with a single-phase supply by installing an external rectifier and a capacitor on the D-AVR mounting plate. In no-load operation, the D-AVR must provide a sufficient field current for the Generator to produce the rated voltage at the rated speed. From no-load to full-load operation, the excitation current must be increased to compensate the effects of the armature reaction. In a generator short circuit, the D-AVR cannot limit the excitation current and all the power fed to the AVR is used in excitation. Thus the excitation current must be limited by other means in order to reduce the generator short-circuit current. This can be done by using a separate excitation current limiter. Voltage and current transformers are needed to measure the instantaneous values of the main voltage and current to enable the voltage regulation. As the load current increases, the armature reaction reduces the air-gap flux and a voltage drop occurs in the terminals of the generator. The AVR must increase the excitation current to compensate the armature reaction and the voltage drops caused by the reactance's of the machine. The microcontroller based automatic voltage regulator. In Microcontroller based Automatic Voltage Regulating system the terminal voltage from the synchronous generator is fed to the dsPIC[8]. The dsPIC30F family of devices includes peripherals that are ideally suited for power control applications. These peripherals include a high-resolution PWM module, a 10-bit 2 Msps Analog-to-Digital Converter module and an Analog Comparator module. The software embedded in the dsPIC calculates the firing angle and fires the thyristor bridge in such a way to maintain the terminal voltage to a referred value. On calculating the firing angle over excitation limit, under excitation limit, power factor and synchronized operation of the generator has to be maintained. Terminal voltage and current is fed to the dsPIC with the help of PT and CT and the power factor is also fed to the IC with the help of the timing circuit. Terminal voltage is stepped down in the range of 0-5v and fed to the IC with the help of zero crossing detectors $[2,4,5,7]$.

\subsection{SYNCHRONISED WORKING OF GENERATOR}

While generator running in parallel with grid the excitation has to be properly matching may lead to undesirable reactive loads which may overload alternator an utility factor become very poor. In case of alternator operating with grid, it has to run at a constant power factor to meet EB requirement. And also to maintain the ratio of active and reactive power irrespective of grid voltage variation to get optimum utilization. The power factor controller sense the power factor of the alternator, compare with the reference set and correct excitation through digital reference unit [DRU].To avoid oscillation in correction, a dead band setting pot is also given to adjust the dead band, depending on the fluctuation of the bus voltage. Two pots are provided to adjust on time of the rise pulse and on time of lower pulse. Power Factor control by using separate power factor controller unit which corrects the excitation parameters through Digital Reference Unit while operating parallel with grid .Follow up unit is provided to follow the auto channel and gives corrective signals to the manual channel. During external fault conditions automatic change over from auto to manual takes place without disturbing the load conditions. After rectification of the fault, change over from manual to auto is possible. That is to change from manual to auto without disturbing the load conditions; manually we have to set the reference of auto channel equal to the manual channel[9]. 


\subsection{UNDER/OVEREXCITED CONDITION OF SYNCHRONOUS GENERATOR}

During generator operation, it is necessary to observe the permissible combinations of active and reactive power, which can be seen from the capability diagram. Limiters employed in the digital excitation system limits the dangerous operation of the generators, which can cause damages as overheating, over fluxing and loss of synchronism. The limiters take the operating point to a state inside of the limits of machine's capability. Limiters can be over excitation limiter, under excitation limiter, overvoltage limiter, over current limiter, voltage/frequency limiter although they can include some others for example the power factor limiter and reactive power limiter. For this application the over excitation limiter, under excitation limiter and $\mathrm{V} / \mathrm{Hz}$ limiter were developed in the DSP.The under excitation limiter corrects the reactive power by raising the machine voltage as necessary to ensure that, in case of an excursion beyond the limit characteristic, the operating point is returned to that characteristic before the machine is tripped by the under excitation protection.

The over excitation limiter ensures that, in the overexcited range, the operating point always keeps within capability curve section of the generator. In response to system voltage drops caused by high reactive power requirements, switching manipulations or faults, the voltage regulator raises the excitation level so as to keep the generator voltage constant. The over excitation limiting device acts as a safeguard against thermal overloading of the rotor.

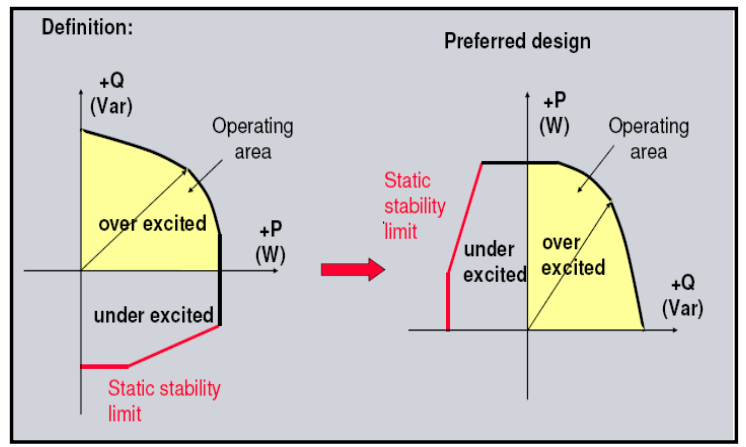

Fig.4. Possible Design of the Generator Capability Diagram

The over excitation limiter admits excitation current values between The maximum continuous current and the maximum excitation Current (field forcing) for a limited period of time so that the Generator can back up the system in response to short-time system Voltage dips. The secondary excitation current limiter (field-forcing limiter), in contrast, has the task of limiting the excitation current to the maximum permissible value as quickly as possible. The stator current limiter ensures the delayed limitation onto working points, within the N-P range of the generator power diagram. The main task of the stator current limiter is to prevent the generator stator from thermical overload, whichcan be caused by a high reactive power at increased active power.

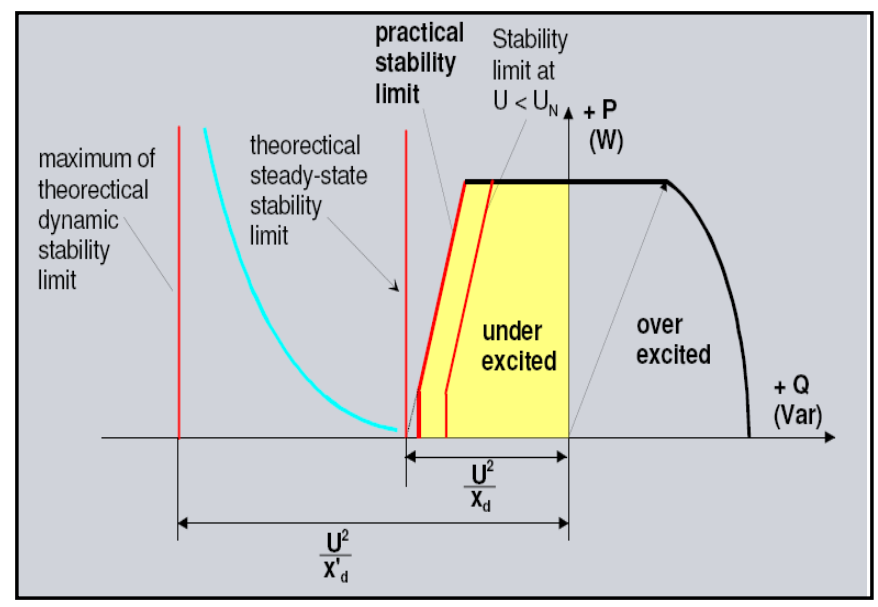

Fig.5. Possible Design of the Generator stability Diagram

The stator current limiter also permits increased excitation values for a limited period so that the generator can back up the system.The over excitation limiter prevents an overheating in the machine's field winding. The under excitation limiter avoids the machine loss of synchronism or avoids an overheating in the stator end region. The volt/Hertz limiter receives the terminal voltage and its frequency as input signals, and protects the synchronous machine and transformers connected to it damages by conditions of over flux. The over flux is caused due to a big relation between voltage and frequency, caused for a suddenly frequency drop. The control of the power factor is also important for the stabilized working of generator.While generator running in parallel with grid or with other alternators the excitation has to be properly matched. Improper matching may lead to undesirable reactive loads which may overload the generator and utility factor becomes very poor. If the power factor is properly balanced to load power factor for alternator running in parallel the utilization of alternator is maximum. In case of alternator operating with grid, it has to run at constant power factor to meet $\mathrm{EB}$ requirement of maintaining the ratio of active and reactive power irrespective of grid voltage to get optimum utilization. The timing unit senses the angle between voltage vector and current vector and this angle is fed to the dsPIC as interrupts.[10] 


\section{FLOWCHART}
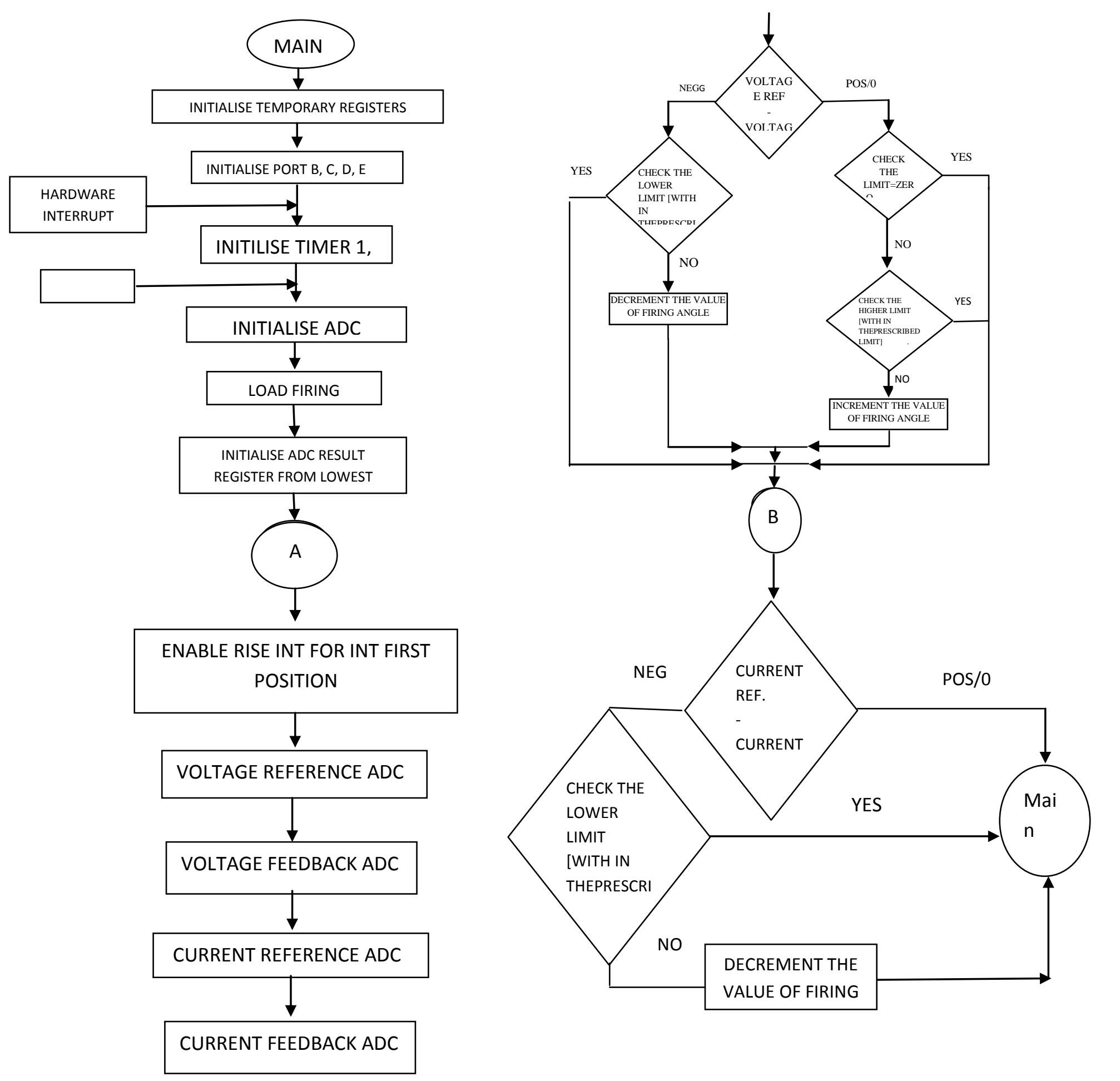


\section{COMPLETE BLOCK DIAGRAM}

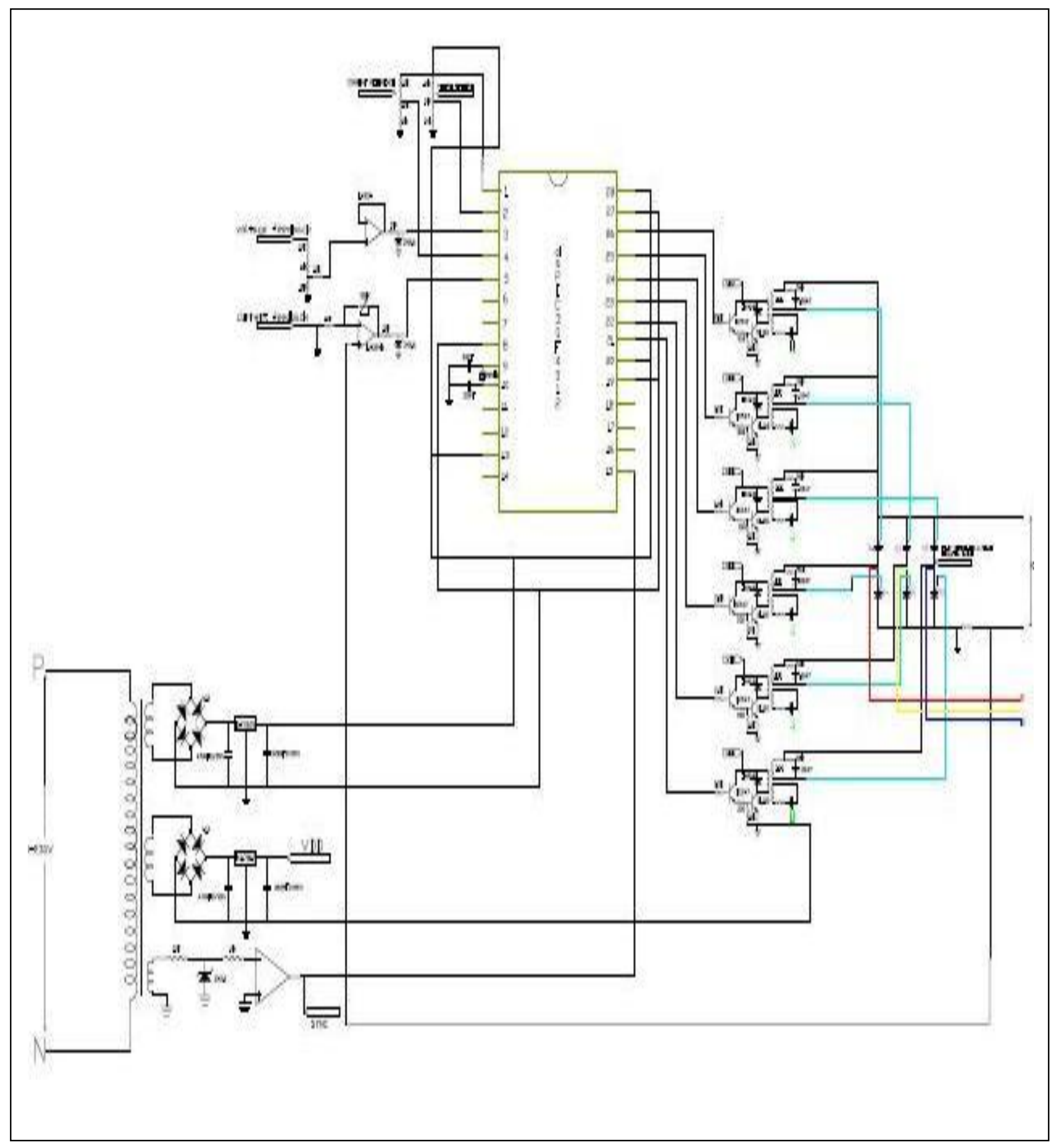




\section{CONCLUSIONS}

The digital excitation system developed by DSPIC. The structure utilized for the task execution allowed a good performance of the system dynamics.

Manual and auto control functions using proportional action and proportional integral action were enough for obtaining fast response speed and a zero state stationary error. The over excitation limiter implemented using a first order dynamics permits that the limiter operates faster when previous over excitation levels are high; on the other hand the hysteresis zone avoids oscillations around the limit point when the limiter is given back to zero.

The under excitation limiter presets a good performance, however oscillations around the operation limit zone were observed, because its structure moved the generator operation point to the limit line. The operation of the $\mathrm{v} / \mathrm{hz}$ limiter was adequate; when is presented a relation between voltage/frequency on top of $1.05 \mathrm{pu}$ decreased the voltage in the same proportion that the frequency drops, avoiding an excessive overflux. Future implementations require to reject the oscillations presented on the under excitation limiter and the overshoots in the voltage/frequency limiter. Thus by employing under excitation as well as over excitation limiters an efficient and a balanced calculation of the firing angle can be obtained from the DSPIC 30F 4012nd fed to the thyristor bridge.

\section{REFERENCES}

[1]JB Gupta, "Theory and performance of Electrical Machines", S.K. Kataria and Sons publishers, Fourteen Edition.

[2]Dr. P. S. Bimbra, "Generalized Theory of Electrical Machines" Khanna publishers, Fifth Edition.

[3]. R.Krishanam, "Electric Motor drives Modelling, analysis and control", PHI-India-2009

[4].Orozco, M. L.; Vasquez, H., "Dynamic performance of an excitation system built in a digital way," Industrial Electronics and Control Applications, 2005. ICIECA 2005

[5] Juan Dixon (Ph.D.), "three phase controlled rectifiers", Department of Electrical EngineeringPontificia Universidad Católica de ChileVicuña Mackenna 4860 Santiago, CHILE.

[6] Prof. Krishna Vasudevan, Prof. G. SridharaRao,Prof. P. SasidharaRao" Electrical Machines II"Indian Institute of Technology Madras.

[7] http://operation of three phase thyristor bridge rectifier service.eng.uts.edu.

[8]http://microchip.com/dspic30f4012/datasheet.

[9] http://WWW.abb.com/motor\&drives.

[10] Dr. Hans-Joachim Herrmann, "Under excitation Protection", Power Transmission and Distribution, Siemens, 2007.

\section{BIOGRAPHIES:}

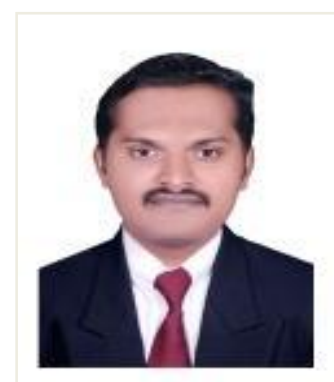

Sreehari $S$ received the B.Tech degree in Electrical \& Electronics Engineering from Calicut University Institute of Engineering \&Technology, under University of Calicut Kerala, India, in 2010 and currently doing M.Tech in Power Electronics in Amrita vishwavidyapeetham, Coimbatore, India. He has published papers in various international Journals. His research interests Multilevel inverter structures, renewable energy technologies, electric drives and control and Excitation systems.

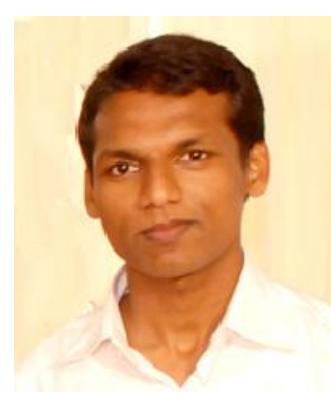

Deepak C $\mathrm{R}$ received the B.Tech degree in computer science from university of Calicut, Kerala, India, in 2011 and currently doing M.Tech in computer vision and image processing in Amrita vishwavidyapeetham university, Coimbatore, India. He has published papers related to image processing and electrical engineering in various international journals. His research interests include content based video/image retrieval, document image analysis,digital image watermarking and computer vision. 